\title{
The responding relationship between plants and environment is the essential principle for agricultural sustainable development on the globe
}

\author{
Zhou Yi ${ }^{\text {a,f,1 }}$, Shao Hong-Bo ${ }^{\text {b,c,d,e,*,1 }}$ \\ a State Key Laboratory of Urban and Regional Ecology, Research Center for Eco-Environmental Sciences, \\ Chinese Academy of Sciences (CAS), Beijing 100085, China \\ ${ }^{\mathrm{b}}$ Binzhou University, Binzhou 256603, China \\ ${ }^{\mathrm{c}}$ Institute of Soil and Water Conservation, Chinese Academy of Sciences \& Water Resources Ministry (CAS\&WRM), Yangling 712100, China \\ ${ }^{\mathrm{d}}$ Institute of Urban Environment, Chinese Academy of Sciences (CAS), Xiamen 361003, China \\ e Institute of Life Sciences, Qingdao University of Science and Technology, Zhengzhou Road 53, Qingdao 266042, China \\ ${ }^{\mathrm{f}}$ Graduate University of Chinese Academy of Sciences (GUCAS), Beijing 100049, China
}

Received 19 December 2007; accepted after revision 17 January 2008

Available online 5 March 2008

Presented by Philippe Morat

\begin{abstract}
The mutual-responding relationship between plants and environment is involved in all life processes, which are the essential bases for different types of sustainable development on the globe, particularly the critical basis for agricultural sustainable development. How to regulate the above relationship between plants and the corresponding environment (in particular soil environment) is the key problem to modern sustainable agriculture development under global climate change, which is one of the hot topics in the field of plant biology. Detailed dissection of this responding relationship is also important for conducting global eco-environmental restoration and construction. Although powerful methodology and dataset related to genomics, post-genomics, and metabolomics have provided some insights into this relationship, crop physiological measures are also critical for crop full performance in field. With the increase of tested plants (including model plants) and development of integrated molecular biology, a complete understanding of the relationship at different scales under biotic and abiotic stresses will be accelerated. In the current paper, we will cover some important aspects in combination with the recent work from our laboratory and related advances reflected by international academic journals, as follows: plant physiological function performance under natural condition, plant gene regulatory network system under abiotic stresses, gene regulatory network system and drought resistance improvement, summary of the related work from our laboratory, conclusions, and acknowledgement. To cite this article: Y. Zhou, H.-B. Shao, C. R. Biologies 331 (2008).
\end{abstract}

๑ 2008 Académie des sciences. Published by Elsevier Masson SAS. All rights reserved.

Keywords: Plants; Gene regulatory network system; Abiotic stress; Signal transduction; Soil environment; Physiological mechanisms

\footnotetext{
* Corresponding author.

E-mail address: shaohongbochu@126.com (H.-B. Shao).

1 Shao Hong-Bo and Zhou Yi is the Co-first author because of equal contribution to this article.
} 


\section{Introduction}

Plants differ from animals in many aspects, but the important may be that plants are more easily influenced by environment than animals [1-4]. Plants have a series of fine mechanisms for responding to environmental changes, which has been established during their long-period evolution and artificial domestication [5-9]. These mechanisms are involved in many aspects of anatomy, physiology, biochemistry, genetics, development, evolution, and molecular biology, in which the adaptive machinery related to molecular biology, is the most important [10-33]. The elucidation of it will extremely and purposefully promote the sustainable utilization of plant resources and make the best use of its current potential at different scales [27,28,34-43]. This molecular mechanism at least includes environmental signal recognition (input), signal transduction (many cascade biochemical reactions are involved in this process), signal output, signal responses and phenotype realization, which is a multi-dimension network system and contains many levels of gene expression and regulation [43-53]. On the basis of our recent work [1-24,42-49] and related publications from other laboratories over the world, we will focus on some important aspects involved in the mutual-responding relationship between plants and environment under abiotic stresses (e.g., drought, salinity, and high temperatures), and draw a possible blueprint for this frontier field between crop biology and plant biology.

\section{Plant physiological function performance under natural condition}

Plants survive in a soil-plant-atmosphere continuum (SPAC) environment, and they have to coordinate the mechanisms of diverse types to respond to the above changing environment at any time for sustainable survival [26-32]. Plant production realization is obtained eventually through physiological pathways at least at the level of the individual and the community [34-39]. One molecule, one kind of tissue or an organ cannot produce any economic yield in terms of need for human beings [40-45,47]. Under the condition of ensuring crop survival, plants can produce the corresponding yield. Water is one of key factors influencing plant production, and many reports have proved this clearly. Loss of water in soil environment will lead to great reduction in crop production, which has been reflected by the total grain yield of many countries in the world [25,30-41]. Water is the important material for photosynthetic reactions that plants depend on to finish accumulation of photosynthetic products, which are impacted greatly by physiological pathways and environmental factors (such as soil water and mineral element supply). Therefore, different soil water supplying will result in quite different physiological pathways, which directly determine the ability for plants to make photosynthetic products. Water deficits in soil environment also influence solute transport (ion and nutrient uptake of plants) to larger extent, which effects on photosynthetic reactions in plant chloroplasts in many ways [27-29]. This is the reason why ion homeostasis and redox state have been brought to attention [26-28,51-53]. The series of the above reactions and processes occurring at different biointerfaces is regulated and controlled spatially and temporally by a crop gene regulatory network system based on the response to crop developmental cues, through which plants can elegantly respond to changes of their environment $[27,28,53]$. This network system has been formed by the interaction between plants and environment for a long time in the process of evolution, and it will continue to evolve with environmental succession $[12,14$, $27,28]$. From the angle of individual plant development, the plant-growth grand periodicity curve can also reflect and show the above-mentioned trend $[4,5,23,32]$. Besides, crop responses to soil water deficits (including nutrients) take a 'slow-fast-slow' shaped curve in terms of main physio-biochemical indices change, and this is in agreement with the plant-growth grand periodicity, which also illustrates this fact and the wide plasticity for plants $[9,23,33,34,53]$. Surely, concerted expression of corresponding genes in the crop gene regulatory network system makes it possible that we can see the phenotype and the phenotype change under given temporalspatial conditions [1-21,33,34,40,53].

\section{Plant gene regulatory network system under abiotic stresses}

Recent progress in molecular biology and bioinformatics (especially, DNA microarray technology), genomics, proteomics, and metabolomics has offered an insight into the crop gene regulatory network system, which is mainly composed of inducible genes (environmental factors and developmental cues), their expression programming, and regulatory elements (ciselement and trans-element), corresponding biochemical pathways, and diverse signal factors [53]. Under the condition of soil water deficits, related stress factors always result in overlapping responses, including anatomical, physiological, biochemical, molecular biological changes, which make the crop gene regulatory network system more complicated and difficult to explore. Much 
Table 1

Typical transcriptional elements related to abiotic stress in higher plants

\begin{tabular}{|c|c|c|}
\hline Plant materials & Responsive factors & Binding sites/Factor types \\
\hline$\overline{\text { Arabidopsis thaliana }}$ & ABI5/AtDPBF & ABA response elements (ABREs)/bZIP \\
\hline A. thaliana & AtDPBF2 & ABA response elements (ABREs)/bZIP \\
\hline A. thaliana & AtDPBF3/AREB3 & ABA response elements (ABREs)/bZIP \\
\hline A. thaliana & AtDPBF4 & ABA response elements (ABREs)/bZIP \\
\hline A. thaliana & AtDPBF5/ABF3 & ABA response elements (ABREs)/bZIP \\
\hline A. thaliana & $\mathrm{ABF} 1$ & ABA response elements (ABREs)/bZIP \\
\hline A. thaliana & ABF2/AREB5 & ABA response elements (ABREs)/bZIP \\
\hline A. thaliana & ABF4/AREB2 & ABA response elements (ABREs)/bZIP \\
\hline A. thaliana & GBF3 & ABA response elements (ABREs)/bZIP \\
\hline A. thaliana & AB53 & $\mathrm{RY} / \mathrm{sph}$ elements/B3 domain proteins \\
\hline A. thaliana & ATMTB2 & MTC \\
\hline A. thaliana & ATHB6 & HD-Zip \\
\hline A. thaliana & ATHB7 & HD-Zip \\
\hline A. thaliana & ATHB12 & HD-Zip \\
\hline A. thaliana & ABI4 & AP2 \\
\hline Oryza & TRAB1 & ABA response elements (ABREs)/bZIP \\
\hline Oryza & OsVPI & $\mathrm{RY} / \mathrm{sph}$ elements/B3 domain proteins \\
\hline Zea mays & VP1 & MYB \\
\hline Triticum & EmBP-1 & ABA response elements (ABREs)/bZIP \\
\hline Avena & AtVPI & $\mathrm{RY} / \mathrm{sph}$ elements/B3 domain proteins \\
\hline Helianthus & DPBF5,-2,-3 & ABA response elements (ABREs)/Bzip \\
\hline Phaseolus & ROM2 (repressor) & ABA response elements (ABREs)/Bzip \\
\hline Phaseolus & PIARF & $\mathrm{RY} / \mathrm{sph}$ elements/B3 domain proteins \\
\hline Craterestinma & Cpvp1 & $\mathrm{RY} / \mathrm{sph}$ elements/B3 domain proteins \\
\hline Daucus & $\mathrm{C}-\mathrm{ABI} 3$ & $\mathrm{RY} / \mathrm{sph}$ elements/B3 domain proteins \\
\hline Populus & PtABI3 & $\mathrm{RY} / \mathrm{sph}$ elements/B3 domain proteins \\
\hline
\end{tabular}

information with respect to this topic is from the model plant Arabidopsis thaliana and rice.

Plants can sense, process, respond to environmental stress, and activate related-gene expression to increase their resistance to stress. Environmental stress-inducible genes can be mainly divided into two types in terms of their protein products:

- a first type, whose coding products directly confer the function of crop cells to resist to environmental stress, such as LEA protein, anti-freezing protein, osmotic regulatory protein, enzymes for synthesizing betaine, proline and other osmoregulators;

- a second type, whose coding products play an important role in regulating gene expression and signal transduction, such as the transcriptional elements for sensing and transducing the protein kinases of MAP and CDP, bZIP, MYB, and others [27-30,39,40,47].

Transcriptional elements are defined as the protein combining with the specialized DNA sequence of eukaryotic promoters or the protein having structural characteristics of known DNA-combining region, whose main function is to activate or suppress the transcrip- tional effect of the corresponding genes. Up to now, hundreds of transcriptional elements of environmental stress-responsive genes in higher plants have been isolated, which regulate and control the stress reaction related to drought, salinity, cold, pathogens, and heat. In the genome of Arabidopsis and rice, there are about 1300-1500 genes for coding transcriptional elements, most of which have not been identified functionally. A recent study has shown that the transcriptional elements involved in crop stress responses mainly include four kinds: APETALA2/EREBP, bZIP, WRKY, and MYB. Typical transcriptional elements have been summarized in Table 1 for reference.

\section{Plant gene regulatory network system and crop drought resistance improvement}

The previous gene engineering strategy for crop stress resistance was to express one (in most cases) or several stress-tolerant genes by constitutive or stressinduced promoters [33,34]. For instance, by introducing betA gene derived from $E$. coli into tobacco and potato, betaine content in the transgenic plants increased to $5 \mu \mathrm{mol} / \mathrm{g}$ (dry weight), and tolerance to salt and cold for the transgenic plants was improved greatly. The 
goal of the recently established plant gene engineering strategy based on transcriptional elements is to improve plant comprehensive-resistance characters (see [24] for detailed references). Compared with the previous traditional method for introducing or improving individual functional genes, the new strategy will play a more important role in crop molecular breeding, because modifying regulatory activities of a transcriptional element can influence functions of many genes at the same time, easily reaching the aim of improving crop comprehensive resistance (to drought, salinity, freezing, diseases, UV-B, and others). Through constitutively overexpressing DERB1A, crop stress-responsive genes, Kin1, cor6.6/Kin2, cor15a, cor47/rd17, ord10 got higher expression and the obtained transgenic Arabidopsis plants were resistant to drought, cold, and salinity. Other related studies also provided a solid evidence of the high efficiency of the above-mentioned methodology.

Dotson (2005) reported that by transferring a transcriptional element into Arabidopsis, which was thought previously not to be related to plant drought response, he obtained many transgenic plants highly resistant to soil water deficits by selecting the Arabidopsis community with higher expression (http://www.int1pag.org/13/abstracts/PAG13-W006.htm1). By further introducing the member of this transcriptional element family into soybean, transgenic soybean lines were cultured and they were resistant to soil water deficits in greenhouse and field. This indicated that the function of this transcriptional element family was characteristic of conservativeness among diverse plant species. Therefore, it is possible to obtain the expected same stressresistant phenotype by genetically modifying transcriptional elements and reach the aim of improving plants efficiently and purposefully [52,53]. Besides, some transcriptional elements not only regulate metabolic pathways, but also influence transport and allocation of secondary metabolites. Plant secondary metabolism plays an important role in plant response to environmental stresses. Long-step progress has taken place in terms of introducing transcriptional elements to regulate targeted pathways of plants.

It is important to remember the fact that some transcriptional elements may regulate several metabolic pathways, and that one metabolic pathway may need orchestrated regulation from some transcriptional elements, which is the nature of the plant (crop) gene regulatory network system. Therefore, in some cases, only introducing a transcriptional element is not enough to obtain the targeted phenotype and may lead to metabolic unbalance in plants. In addition, because of coordi- nated evolution of transcriptional elements and of their regulating metabolic pathways, the genetically modifying strategy for the same transcriptional element could produce different phenotypes in different crop species. These issues need deeper exploration to establish an efficient genetically modifying system by transcriptional elements and their network system for improving crop stress resistance and global eco-environment and feeding the world [2,14,33,34,48-53].

In a word, precise elucidation of the plant gene regulatory network system under abiotic stress is of importance to molecularly engineering crop resistance, because of which many excellent scientists worldwide have been engaged in this frontier field, resulting in a long-step progress. There are also many issues that remain to be solved and need efforts to be made. The scope of tested plants needs to be extended; a comprehensive study of the combination of environmental stress factors in laboratories and in the field should be given much attention; system developmental viewpoints and computer simulation analysis should be also applied. With the accumulation of data from an extended range of plants (crops), the crop gene regulatory network system under environmental stresses will be clearer and clearer (Fig. 1).

\section{Summary of the related work from our laboratory and its extending implications}

First, our group applied a barley matured embryo in vitro system to investigate the widely used osmotic regulator, PEG-6000's effect on hormone content (ABA, GAs, IAA) in barley seeds, embryos, endosperm, and regenerated plantlets. We showed that different ions $\left(\mathrm{Ca}^{2+}, \mathrm{Mg}^{2+}, \mathrm{Na}^{+}, \mathrm{K}^{+}, \mathrm{Fe}^{2+}, \mathrm{Zn}^{2+}\right)$ in culture media had different influences upon plant growth and development. This conclusion is of great help to understand substance exchange between the soil and the plant root system, in particular nutrients at soil water deficits. The different ions in the soil environment have obvious effects on physiological functions of upper crop parts [1].

Second, the LEA (late embryogenesis abundant) protein is one type of important proteins mainly involved in crop drought resistance and development. Our group gave a good review about this hot topic based on related work [2-6], which will provide a convenient guide to conduct a corresponding plant molecular biology and agricultural biotechnology study [2].

Third, the most important work from our laboratory is the contribution to crop stress biology. My group used 10 different wheat genotypes cultured by the Yangling 


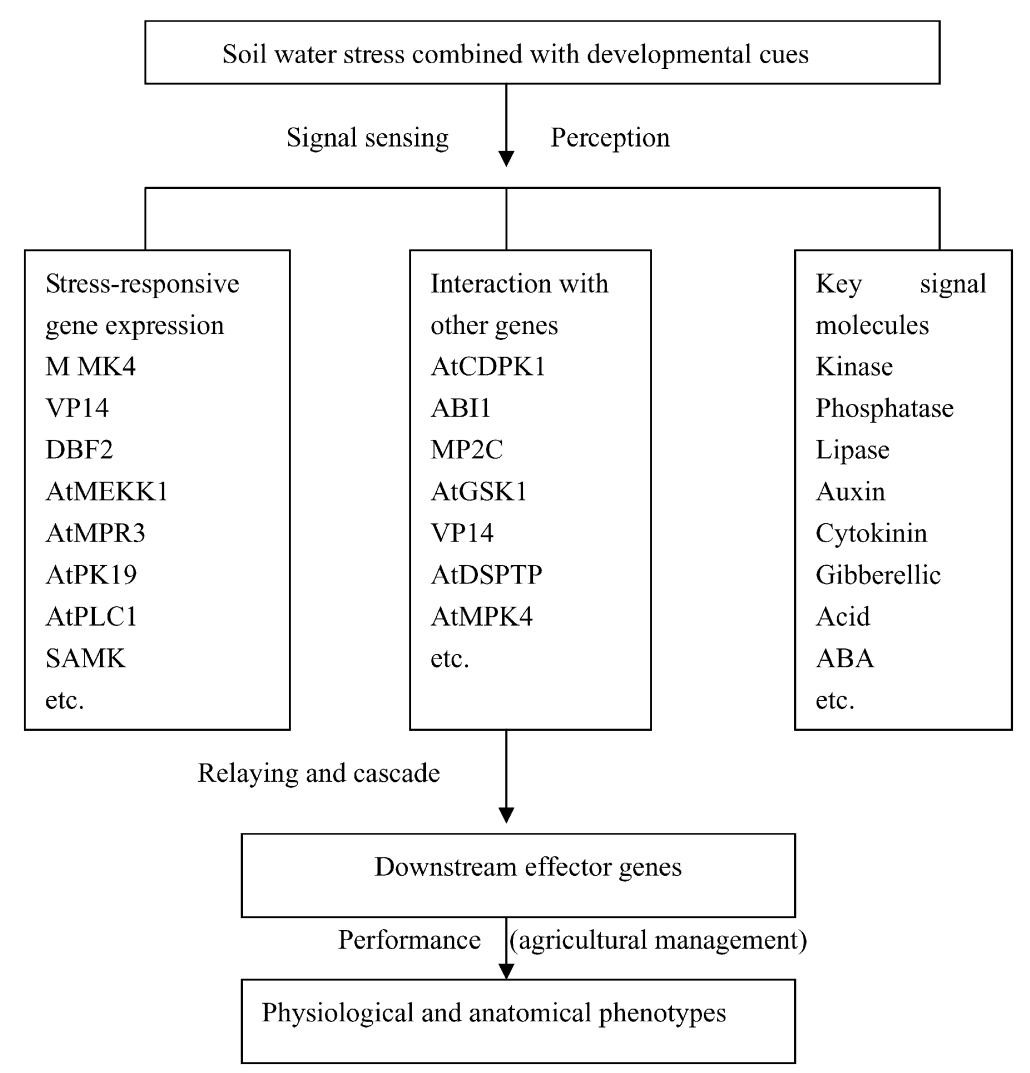

Fig. 1. The basic draft for the plant gene regulatory network system $[14,23]$.

National Wheat Breeding Centre of China to do a series of work involved in crop stress physiology, which has set up a bridge between soil science and crop physiology, molecular biology and biotechnology. From the angle of development (seedling, tillering, and maturation stages), my group explored the dynamic change of POD, SOD, CAT and MDA among 10 wheat genotypes at soil water deficits $[3-7,15,43]$. We concluded that the changing law of these anti-stress indicators took a tendency of 'slow-rapid-slow' curve, which is in agreement with the curve of plant growth periodicity coincidently. This viewpoint brought out the plastic responses of plants to stress environments, which is of great importance to understand deeply crop speciation and diversity evolution $[48,49]$. The above work also helps conduct global dryland farming, water-saving agriculture, and vegetation restoration practice.

Fourth, from the aspect of different growth-development stages, related investigation of wheat photosynthetic physiology is very much limited. Dr. Shao's group used the above experimental materials to study the dynamic photosynthetic change under the condition of soil water deficits. They showed that photosynthetic efficiency (Pn), transpiration rate (Tr) and stomatal con- ductance (Cond) of 10 wheat genotypes under the condition of three soil water stress levels were very much related to wheat genotypes and to their corresponding cultivation zones. Intercellular $\mathrm{CO}_{2}$ concentration $\left(C_{i}\right)$ was not when it reached a certain concentration [5]. This discovery is the first report in crop physiology, and it is helpful to evaluate photosynthetic functions of different crop species finely. The above four parameters are important indicators for measuring photosynthetic efficiency, but their roles in this aspect cannot be considered the same. This discovery also has an importance in conducting global precise agriculture and high production of plants [9-17].

\section{Conclusions}

In a word, the core issue for agricultural sustainable development and ecoenvironmental construction is to coordinate well the responding relationship between plants (plants) and the environment at different scales $[44,52,53]$. Up to date, much information from the scale of plant biology is available and successfully applied in traditional agriculture, but to solve the above problem and feed the globe under climate change and 
the pressure of other serious issues, more information from other scales of biology is needed, which is the main challenge for the human being in the 21 st century. A complete dissecting of the mutual-responding relationship at different levels of plant biology is critical for modern agriculture sustainable development and precise agriculture, but many problems remain to be resolved. Our group has done some primary work in crop stress biology that has basically established an efficient platform for crop stress molecular biology and biotechnology. Of course, our work also need much work of field and water cultivation experiments to make complements. In addition to continuing to explore the mutual-responding relationship between plants and environment by physiological and biochemical methodology, the future work in our laboratory will focus on the integrated physiological law of plants under abiotic stress for making the best use of crop physiological potential for optimum productivity, and partial work will be transferred to the role of main antioxidants and redox signalling in plants. Some important work needs be elucidated as follows. Besides exacerbating cellular damage, reactive oxygen species (ROS) can act as ubiquitous signal molecules in plants. ROS are a central component in stress responses and the level of ROS determines the type of response. Antioxidants and ROS are an important interacting system with different functions in higher plants, which ensures to them a highly flexible organism. Redox signal transduction is a universal characteristic of aerobic life honed through evolution under natural and selecting pressure to balance information from metabolism and the changing environment. Crop cells can be considered as a series of interconnecting compartments with different antioxidant buffering capacities, determined by differences in synthesis, transport, and/or degradation. There is a set of discrete locations where signalling is controlled (or buffered) independently in higher plant cells, which permits redox-sensitive signal transduction to occur in locations such as the apoplast, the thylakoid, and the endoplasmic reticulum, whereas other highly buffered spaces have a much higher threshold for ROS signals [25-28]. Both oxidants and antioxidants fulfil signalling roles to provide information on higher plant health, particularly in terms of robustness for defence, using kinase-dependent and in-dependent pathways that are initiated by redox-sensitive receptors modulated by thiol status. Antioxidants are not passive bystanders in this crosstalk, but they rather function as key signalling components that constitute a dynamic metabolic interface between higher plant (crop) cell stress perception and physiological responses [38-41,53]. There can be no doubt that transgenic plants will be invaluable in assessing the precise role that main antioxidants and ROS play in the functional gene network that controls crop stress tolerance. Although steps in the biosynthetic pathways resulting in antioxidant accumulation in higher plants have been primarily characterized at the physiological and molecular levels, the full cast of participants involved in the complex regulation of their accumulation remains to be identified. This will require not only a better understanding of the degradation and transport of antioxidants in higher plants (plants), but also elucidation of the molecular events responsible for stress perception and stress-related signal transduction via wider scope of tested plants (plants). Basic research leading to the characterization of tightly regulated stress-inducible promoters that are also responsive to appropriate tissue-specific regulation and endogenous developmental programmes is likely to be critical in improving the overall field performance of transgenic plants [49-53]. The future will determine more precisely how ascorbate, glutathione, and tocopherol are involved in initiating and controlling redox signal transduction and how they trigger the gene expression of other related responses to optimize crop survival strategies. In addition, other problems are how antioxidants coordinate growth and development of plants in a constantly changing environment, how redox signalling is linked with hormonal regulation, nutrient status and redox potential of plants, and how their redox signalling is cooperated with inter- and intracellular signalling, transport capacity, developmental and environmental cues to maintain an appropriate dynamic homeostasis for crop stress tolerance and its efficient survival.

\section{Acknowledgements}

Research in Professor Shao H.B.'s laboratory is jointly supported by 973 Program of China (2007CB106803) and the Shao Ming-An's Innovation Team Projects of the Education Ministry of China and Northwest A\&F University.

\section{References}

[1] H.B. Shao, Z.S. Liang, M.A. Shao, B.C. Wang, Impacts of PEG6000 pretreatment for barley (Hordeum vuldare L.) seeds on the effect of their matured embryo in vitro culture and primary investigation on its physiological mechanism, Biointerfaces 41 (2-3) (2005) 73-77.

[2] H.B. Shao, Z.S. Liang, M.A. Shao, LEA protein in higher plants: structure, functions and gene expression regulation, Biointerfaces 45 (3-4) (2005) 131-135. 
[3] H.B. Shao, Z.S. Liang, M.A. Shao, B.C. Wang, Changes of some physiological and biochemical indices for soil water deficits among 10 wheat genotypes at seedling stage, Biointerfaces 42 (2) (2005) 107-113.

[4] H.B. Shao, Z.S. Liang, M.A. Shao, Dynamic changes of antioxidative enzymes of 10 wheat genotypes at soil water deficits, Biointerfaces 42 (3-4) (2005) 187-195.

[5] H.B. Shao, Z.S. Liang, M.A. Shao, Investigations on dynamic changes of photosynthetic characteristics of 10 wheat genotypes during two vegetative-growth stages at water deficits, Biointerfaces 42 (2) (2005) 221-227.

[6] H.B. Shao, Z.S. Liang, M.A. Shao, Changes of anti-oxidative enzymes of 10 wheat genotypes at maturation under the condition of soil water deficits, Biointerfaces 45 (1) (2005) 7-13.

[7] H.B. Shao, L.Y. Chu, G. Wu, J.H. Zhang, Z.H. Lu, Y.C. Hu, Changes of some anti-oxidative physiological indices under soil water deficits among 10 wheat (Triticum aestivum L.) genotypes at tillering stage, Biointerfaces 54 (2) (2007) 143-149.

[8] H.B. Shao, Z.S. Liang, M.A. Shao, Adaptation of higher plants to environmental stresses and stress signal transduction, Acta Ecol. Sin. 25 (7) (2005) 1771-1782.

[9] G. Wu, Z.K. Wei, H.B. Shao, et al., The mutual responses of higher plants to environment: Physiological and microbiological aspects, Biointerfaces 59 (2007) 113-119.

[10] H.B. Shao, L.Y. Chu , C.A. Jaleel, Water-deficit stress-induced anatomical changes in higher plants, C. R. Biol. 331 (3) (2008) 215-225.

[11] H.B. Shao, X.Y. Chen, L.Y. Chu, X.N. Zhao, G. Wu, Y.B. Yuan, C.X. Zhao, Z.M. Hu, Investigation on the relationship of proline with wheat anti-drought under soil water deficits, Biointerfaces 53 (2) (2006) 113-119.

[12] H.B. Shao, Q.J. Guo, L.Y. Chu, X.N. Zhao, Z.L. Su, Y.C. Hu, J.F. Cheng, Understanding the molecular mechanism of higher plant plasticity under abiotic stress Biointerfaces 54 (1) (2007) 37-45.

[13] H.B. Shao, S.Y. Jiang, F.M. Li, L.Y. Chu, C.X. Zhao, M.A. Shao, X.N. Zhao, F. Li, Some advances in plant stress physiology and their implications in the systems biology era, Biointerfaces 54 (1) (2007) 33-36.

[14] H.B. Shao, L.Y. Chu, C.X. Zhao, Q.J. Guo, X.A. Liu, J.-M. Ribaut, Plant gene regulatory network system under abiotic stress, Acta Biol. Sezeged 50 (1-2) (2006) 1-9 (invited review).

[15] H.D. Chen, V.J. Karplus, H. Ma, X.W. Deng, Plant biology research comes of age in China, Plant Cell 18 (11) (2006) 28552864.

[16] H.B. Shao, L.Y. Chu, G. Wu, J.H. Zhang, Z.H. Lu, Where is the road to bio-watersaving for the globe? Biointerfaces 55 (2) (2007) 251-255.

[17] H.X. Cao, Z.B. Zhang, P. Xu, L.Y. Chu, H.B. Shao, Z.H. Lu, J.H. Liu, Mutual physiological genetic mechanism of plant high water use efficiency and nutrition, Biointerfaces 54 (3) (2007) 213-219.

[18] Z.S. Liang, J.W. Yang, H.B. Shao, R.L. Han, Investigation on water consumption characteristics and water use efficiency of Poplar under soil water deficits on the Loess Plateau, Biointerfaces 53 (1) (2006) 23-28.

[19] Y. Tan, Z.S. Liang, H.B. Shao, F. Du, Effect of water deficits on the activity of anti-oxidative enzymes and osmoregulation among three different genotypes of Radix Astragali at seeding stage, Biointerfaces 49 (1) (2006) 60-65.

[20] Z.B. Zhang, H.B. Shao, P. Xu, L.Y. Chu, Z.H. Lu, J.Y. Tian, On evolution and perspectives of bio-watersaving, Biointerfaces 55 (3) (2007) 291-299.
[21] H. Zhao, Z.B. Zhang, H.B. Shao, P. Xu, M.J. Foulkes, Genetic correlation and path analysis of transpiration efficiency for wheat flag leaves, Environ. Exp. Bot. 62 (3) (2008), in press.

[22] F. Du, H.B. Shao, L. Shan, Z.S. Liang, M.A. Shao, Effects of secondary succession on soil moisture and nutrition in the abandoned old-field of hilly region of Loess plateau, China, Biointerfaces 58 (2007) 278-285.

[23] G. Wu, C. Zhang, L.Y. Chu, H.B. Shao, Responses of higher plants to abiotic stresses and agricultural sustainable development, J. Plant Interactions 2 (3) (2007) 135-147 (invited review).

[24] D. Bartels, R. Sunkar, Drought and salt tolerance in plants, Crit. Rev. Plant Sci. 24 (2007) 23-58.

[25] J.M. Li, H. Jin, Regulation of brassinosteroid signaling, Trends Plant Sci. 12 (1) (2007) 37-41.

[26] L. Pourcel, J.-M. Routaboul, V. Cheynier, et al., Flavonoid oxidation in plants: From biochemical properties to physiological functions, Trends Plant Sci. 12 (1) (2007) 29-36.

[27] C.H. Foyer, G. Noctor, Redox sensing and signaling associated with reactive oxygen in chloroplasts, peroxisomes and mitochondria, Physiol. Plant. 119 (2003) 355-364.

[28] C.H. Foyer, G. Noctor, Redox homeostis and antioxidant signalling: A metabolic interface between stress perception and physiological responses, Plant Cell 17 (2005) 1866-1875.

[29] R. Mittler, Abiotic stress, the field environment and stress combination, Trends Plant Sci. 11 (1) (2006) 15-19.

[30] P.J. Gregory, Roots, rhizosphere and soils: The route to a better understanding of soil science? Eur. J. Soil Sci. 57 (1) (2006) 212.

[31] P. Hinsinger, G.R. Gobran, P.J. Gregory, et al., Rhizosphere geometry and heterogeneity arising from root-mediated physical and chemical processes, New Phytol. 168 (2005) 293-303.

[32] P. Hinsinger, C. Plassard, C. Tang, B. Jaillard, Origins of rootinduced $\mathrm{pH}$ changes in the rhizosphere and their responses to environmental constraints: A review, Plant Soil 248 (2003) 4359.

[33] É De La Barrera, J.-L. Andrade, Challenges to plant megadiversity: how environmental physiology can help, New Phytol. 167 (2005) 5-8.

[34] G.T. How, A.M. Brunner, An evolving approach to understanding plant adaptation, New Phytol. 167 (1) (2005) 1-5.

[35] H. Nakagami, A. Pitzschke, H. Hirt, Emerging MAP kinase pathways in plant stress signaling, Trends Plant. Sci. 10 (7) (2005) 339-346.

[36] M. Boudsocq, C. Lauriere, Osmotic signaling in plants. Multiple pathways mediated by emerging kinase families, Plant Physiol. 138 (2005) 1185-1194.

[37] N. Suzuki, L. Rizhsky, H.J. Liang, J. Shuman, R. Mittler, Enhanced tolerance to environmental stress in transgenic plants expressing the transcriptional coactivator multiprotein Bridging Factor 1c (MBF1C), Plant Physiol. 139 (2005) 1313-1322.

[38] R. Vera-Estrella, B.J. Barkla, L. Garcia-Ramirez, O. Pantoja, Salt stress in Thellungiella halophia activates $\mathrm{Na}^{+}$transport mechanisms required for salinity tolerance, Plant Physiol. 139 (2005) 1507-1517.

[39] H.B. Shao, L.Y. Chu, Plant molecular biology in China: Opportunities and challenges, Plant Mol. Biol. Rep. 23 (4) (2005) 345-358.

[40] Y.C. Hu, H.B. Shao, L.Y. Chu, G. Wu, Relationship between water use efficiency (WUE) and production of different wheat genotypes at soil water deficit, Biointerfaces 53 (3) (2006) 271277. 
[41] H.B. Shao, L.Y. Chu, Z.H. Lu, C.M. Kang, Primary oxidant scavenging and redox signaling in higher plants, Int. J. Biol. Sci. 4 (1) (2008) 12-17.

[42] G. Wu, L.Y. Chu, H.B. Shao, J.W. Cai, Insights into molecular mechanisms of mutual effect between plants and the environment, A review, Agron. Sustain. Dev. 27 (1) (2007) 1-10 (invited review)

[43] G. Wu, P. Jiang, J. Wei, H.B. Shao, Nutrients and biomass spatial patterns in alpine tundra ecosystem on Changbai mountains, northeast China, Biointerfaces 60 (2007) 250-257.

[44] L.Y. Chu, H.B. Shao, Dryland farming and agricultural sustainable development, in: R.A. Lopez (Ed.), Progress in Sustainable Development Research, Nova Science Publishers, Inc., New York, USA, 2007, pp. 117-144.

[45] H.B. Shao, L.Y. Chu, G. Wu, Physiological and molecular responses of higher plants to abiotic stresses, in: N.A. Khan, S. Singh (Eds.), Abiotic Stress and Plant Responses, I.K. International Publishing, New Delhi, India, 2007.

[46] A.H. Millar, V. Mittova, G. Kiddle, Control of ascorbate synthesis by respiration and its implications for stress responses, Plant Physiol. 133 (2003) 443-447.
[47] W.X. Wang, B. Vinocur, A. Altman, Plant responses to drought, salinity and extreme temperatures: Towards genetic engineering for stress tolerance, Planta 218 (2003) 1-14.

[48] M.M. Chaves, J. Maroco, J. Pereira, Understanding plant responses to drought - from genes to the whole plant, Funct. Plant. Biol. 30 (2003) 239-264.

[49] C. Stohr, S. Stremlau, Formation and possible roles of nitric oxide in plant roots, J. Exp. Bot. 57 (3) (2006) 463-470.

[50] P.M. Mullineaux, S. Karpiniski, N.R. Baker, Spatial dependence for hydrogen peroxide-directed signaling in light-stressed plants, Plant Physiol. 141 (2006) 346-350.

[51] B. Halliwell, Reactive species and antioxidants, Redox biology is fundamental theme of aerobic life, Plant Physiol. 141 (2006) 312-322.

[52] H.B. Shao, Z.S. Liang, M.A. Shao, G. Wu, Wheat anti-drought biology: Significance, progress and perspectives, in: C.L. Bai (Ed.), A Collection of Papers from the Winner for Excellent PhD Dissertation of Chinese Academy of Sciences, Science Press, Beijing, China, 2006, pp. 319-329 (in Chinese).

[53] H.B. Shao, L.Y. Chu, C.X. Zhao, Advances in functional regulation mechanisms of plant aquaporins: their diversity, gene expression, localization, structure and roles in plant water relations, Mol. Membr. Biol. 25 (2) (2008), in press. 